\title{
Olfactory Outcome After Endoscopic Sinus Surgery in Chronic Rhinosinusitis
}

\author{
Satish HS ${ }^{1}$, Roopa Malali², Rinki J Pomson ${ }^{3}$, Deepika TM ${ }^{3}$, Athira Thampan ${ }^{3}$ and Borlingegowda Viswanatha ${ }^{4}$ \\ ${ }^{1}$ Professor, Department of ENT, Bangalore Medical College\& Research Institute, India
}

${ }^{2}$ Senior Resident, Department of ENT, Bangalore Medical College\& Research Institute, India

${ }^{3}$ Postgraduate, Department of ENT, Bangalore Medical College\& Research Institute, India

${ }^{4}$ Professor and Head, Department of ENT, Bangalore Medical College\& Research Institute, India

*Corresponding author: Roopa Malali, Senior Resident, Department of ENT, Bangalore Medical College\& Research Institute, Bangalore, India

\begin{abstract}
Background: Olfactory dysfunction is one of the major symptoms of CRS. Olfactory dysfunction affects the quality of life. Endoscopic sinus surgery is the surgical modality of treatment in cases of failed medial therapy. Assessment of olfaction in patients undergoing endoscopic sinus surgery is essential to know the impact on olfaction.

Objective: To assess the olfaction before and after Endoscopic sinus surgery.

Methods: This is a prospective study. Total 45 Patients were included in this study based on the inclusion and exclusion criteria. Diagnostic nasal endoscopy and CT scan were done preoperatively. Olfaction was assessed in all patients preoperatively, postoperatively at 1 month and 3 months using olfactory testing tool.

Results: There were 45 patients of both gender $57.78 \%$ were females and $42.22 \%$ were males. The mean age of patients in our study was found to be $39.82 \pm 6.264$ years. Most of these patients were in fourth decade of life. Of 45 patients $75 \%$ had symptoms of hyposmia/anosmia before surgery. Average Lund Mackey score for CT scan findings was found to be $13.42 \pm 3.1$. The patients were assessed postoperatively at 1 st month and 3rd month using olfactory testing tool. The mean composite olfactory score was $5.83 \pm 2.89$ preoperatively increased to $8.28 \pm 2.33$ at 1 month and $10.12 \pm 1.11$ at 3 months, this difference was found to be statistically significant $(\mathrm{p}<0.001)$. All patients mean olfactory scores improved postoperatively. The mean composite olfactory scores improved following endoscopic sinus surgery by 3.5 in Anosmic patients, 2.22 in Hyposmic patients and 1.04 in Normosmic at 1 month. Whereas at 3 months, 6.65, 3.22 and 1.95 in Anosmic, Hyposmic and Normosmic patients, respectively. The study was an attempt to assess the olfaction preoperatively and postoperatively in patients undergoing ESS.

Conclusion: The evaluation of olfaction in patients undergoing endoscopic sinus surgery showed improvement in olfactory outcome following the surgery in patients with CRS. 61\% to 69\% of CRS patients have a reduced sense of smell. Approximately two thirds of cases are due to the sinonasal disease. Olfactory dysfunction as result of sinonasal disease is unlikely to improve spontaneously without treatment.
\end{abstract}

Keywords: Olfaction; endoscopic sinus surgery; chronic rhino sinusitis

Abbreviations: CRS: Chronic Rhinosinusitis; ESS: Endoscopic Sinus Surgery; CCCRC: Connecticut Chemosensory Clinical Research Centre 


\section{Introduction}

Olfaction is a special sense of smell, which is perceived in the olfactory region of the nasal cavity and helps in enjoying the taste of food. The anatomic state of nasal epithelium and the status of the peripheral and central nervous system determine the quality and the intensity of perception [1]. The perception of odors adds a quality to life. Odors are a part of our everyday life, from pleasures of perfume to satisfaction of toast and coffee [2]. Olfaction survives many purposes from detection of hazards, sexual behavior, flavors of food. The olfactory system impairment has got significant impact on quality of life. The people presenting with olfactory disturbances has got changes in appetite or body weight and also affects psychological well-being. Significant psychological disruption and feeling of physical and social vulnerability occur by the olfactory disturbances. Anosmia is an inability to detect olfactory sensations while hyposmia is reduced ability to perceive odors [2].

Chronic rhinosinusitis (CRS) related olfactory dysfunction is common with prevalence ranging from $48 \%$ to $83 \%$.It is known that medical therapy improves olfactory dysfunction in CRS. For patients failing medical therapy, endoscopic sinus surgery is performed. Addressing the patients with olfactory impairment is important, as it affects the quality of life. Endoscopic sinus surgery (ESS) is the preservation of existing structures by performing limited surgery [3]. ESS is widely used in chronic rhinosinusitis and nasal polyposis. The primary objective of Endoscopic Sinus Surgery is to restore paranasal sinus function by reestablishing the physiological pattern of ventilation and mucociliary clearance [4]. Olfactory disturbances is one of the complications of sinus surgery. Kimmelman tested 93 patients before and after sinus surgery, $34 \%$ patients had decreased olfactory ability [5]. As olfactory dysfunction is difficult to express by patients. Objective assessment of olfactory disturbance is necessary to know outcome of ESS on olfaction. Connecticut Chemosensory Clinical Research Centre Olfactory (CCCRC) test which is composed of threshold testing and odor identification test, it is done to know sinus surgery impact on olfaction [6,7]. Hence, there is a need for study to know the impact of ESS on olfaction.

\section{Objectives}

To assess the olfaction before and after ESS.

\section{Methodology}

\section{Materials and Methods}

Study population and place of study: Patients qualifying and selected for ESS attending the otorhinolaryngological outpatient department (OPD) attached to Bangalore Medical College and Research Institute, Bangalore.

\section{Methods of collection of data:}

Study design: Prospective study

Study period: November 2017 to May 2019.

Sample size: 45 cases

\section{Inclusion criteria}

a) Patients aged $18-50$ yrs.

b) Patients undergoing endoscopic sinus surgery.

c) Patients willing to give written informed consent.

\section{Exclusion criteria}

a) Patients with history of previous Sino nasal surgery.

b) Patients with history of head injury.

c) Patients with deviated septum.

d) History of smoking.

e) History of asthma.

f) Pregnant women.

g) Patients with Atrophic rhinitis.

h) Patients with Parkinsonism disease.

i) Patients not willing to give written and informed consent.

Patients who fulfill the inclusion criteria were enrolled in the study. Written informed consent from patients was taken. Demographic data, detailed history suggestive of chronic rhinosinusitis, nasal polyposis like nasal congestion, headache, facial pain, mouth breathing, anosmia, hyposmia, nasal obstruction and nasal discharge and other relevant history were recorded. Later clinical and otorhinolaryngological examination was carried out. Patients had been explained about procedure clearly. Patients were seated comfortably and blindfolded. The tests were done in a well-ventilated and quiet room. The opposite nostril was plugged with cotton up to the inferior turbinate level. Then patients were subjected to odor identification and odor threshold testing. For odour identification testing, test odorants like baby powder, chocolate, cinnamon, coffee, and soap, and distracters like garlic, ketchup, onion, wood shavings and tobacco will be used. The substances (color coded) will be kept in glass tubes, the uncapped tubes will be placed approximately $1 \mathrm{~cm}$ from the nostril for 3 seconds. The patient should be told to sniff normally and identify the odor presented. Two chances will be given to identify one odor. Patient should identify the four randomly offered odors. The interval of 30seconds is given for successive presentation. The correctly identified odors will be taken as odor identification score of that nostril. Final odor identification score of an individual is calculated by combining the score of both the nostrils. 
For odor threshold testing, test odorants like butanol-1, peppermint oil, clove oil, lemon oil will be used. The test uses an aqueous dilution series of butanol, in which highest concentration $4 \%$ labeled as solution 1 and lowest being solution 9. The successive dilution of butanol differs by a factor 3.The sterile water will be taken as test control. The test will be started with lowest concentration. The patient will be given two glass tubes at a time, one with sterile water, other with odorant (solution 1-9). Then patient asked to identify the tube that smells stronger. Four consecutive correct responses will lead to the termination of test. If response is incorrect, the next higher concentration will be given after a gap of 15 seconds. The patient will be allowed to answer only once. The correctly identified solution take as threshold values 1 to 7 (identification of solution 7, 8,9 commonly assigned as 7). Zero is taken when patient fails to identify the solution 9. The threshold values in the nostril will be added to get odor threshold score of an individual. Trigeminal response was tested with Vicks. These tests are done preoperatively and postoperatively at 1st month and 3rd month after endoscopic surgery.

\section{Statistical Analysis}

The data was entered in an excel spreadsheet and analyzed using IBM SPSS version 20. The descriptive statistics were presented as mean and standard deviation for the quantitative variables whereas in numbers and percentages for qualitative variables. Population pyramid was used to represent age distribution according to sex and pie chart was used represent the distribution of subjects according to presenting symptoms. The olfactory scores were compared preoperatively with postoperatively at 1 month, preoperatively and postoperatively at 3 months using the Wilcoxan Signed Rank test. The mean olfactory scores of preoperative period, postoperatively at 1 month and 3 months were compared using Friedman test. The results were considered significant at $\mathrm{p}$ value of 0.05 (two-tailed).

\section{Results}

There were 45 patients in the study. The mean age of the patients in our study was found to be $39.82( \pm 6.2)$ years. Most of patients were in the $4^{\text {th }}$ decade. Female patients predominate in our study with average of $57.78 \%$ percentage. Of 45 cases, $37.78 \%$ were presented with anosmia, hyposmia in $35.56 \%$ and Normosmia in $24.44 \%$ (Figures 1 \& 2). DNE findings are nasal mucosal edema, nasal discharge, and polyposis. These are graded as per Lund and Kennedy scoring. On right side, Nasal mucosal edema was mild in $53.33 \%$ cases and severe in $44.44 \%$ cases. Nasal discharge was thin \& non purulent in $71.11 \%$ cases and thick \&purulent $13.33 \%$. Polyposis was present in $46.67 \%$. On left side, Nasal mucosal edema was mild in $37.78 \%$ and severe $57.78 \%$. Nasal discharge was thin and non-purulent in $57.78 \%$ and Thick\& purulent in $37.78 \%$. In this study, nasal endoscopy score mean \pm SD $\quad 6.73 \pm 2.562$. Out of 45 patients $57.11 \%$ presented with polyp. $11.11 \%$ being unilateral Ethmoidal polyp, $40 \%$ bilateral Ethmoidal polyp. Preoperative CT scan was done in all patients. Average Lund Mackey score for CT scan finding was found to be $13.42 \pm 3.1$ (Tables $1 \& 2$ ). In our study, olfactory scores are compared preoperatively and postoperatively at 1 month and 3month both sides (right and left). It showed that score improved from $5.91 \pm 3.21$ to $8.33 \pm 2.41$ at 1 month and $5.91 \pm 3.21$ to $10.24 \pm 1.13$ which is statistically significant $(<0.001)$. In our study, the preoperative mean olfactory score was 5.83, which improved to 8.28 and 10.12 in the postoperative period at $1^{\text {st }}$ month and $3^{\text {rd }}$ month, respectively. This was found to be statistically significant (Friedman test and $\mathrm{p}<0.001$ ) (Table 3).

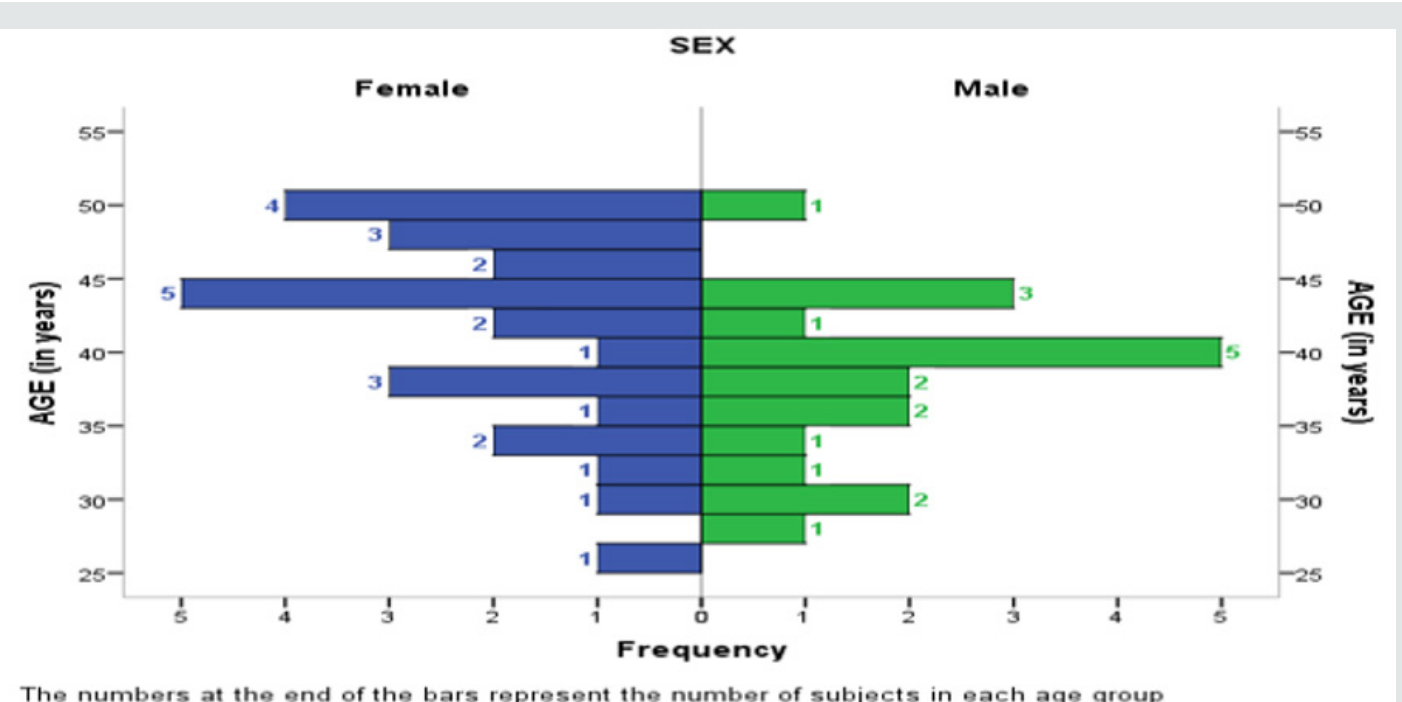

Figure 1: Distribution of study subjects according to age and sex $(\mathrm{N}=45)$. 


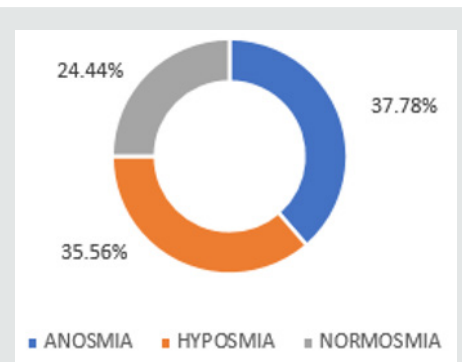

Figure 2: Distribution of study subjects according to presenting symptoms ( $N=45)$.

Table 1: Olfactory score comparison: preoperatively, 1 month and 3 months postoperatively (Right side) $(\mathrm{N}=45)$.

\begin{tabular}{|c|c|c|c|c|c|c|c|}
\hline \multirow{2}{*}{ Score } & \multicolumn{2}{|c|}{ Preop } & \multicolumn{2}{c|}{ Postop 1 months } & \multicolumn{2}{c|}{ Postop 3 months } & P Value \\
\cline { 2 - 8 } & Mean \pm SD & $\mathbf{9 5 \%}$ CI & Mean \pm SD & $\mathbf{9 5 \%}$ CI & Mean \pm SD & 95\% CI & $4.39-4.76$ \\
\hline OIS & $2.56 \pm 1.63$ & $2.07-3.05$ & $3.76 \pm 1.09$ & $3.43-4.08$ & $4.58 \pm 0.62$ & $5.39-5.95$ \\
\hline OTS & $3.36 \pm 1.71$ & $2.84-3.87$ & $4.6 \pm 1.56$ & $4.13-5.07$ & $5.67 \pm 0.93$ & $<0.001$ \\
\hline COS & $5.91 \pm 3.21$ & $4.95-6.88$ & $8.33 \pm 2.41$ & $7.61-9.06$ & $10.24 \pm 1.13$ & $9.9-10.58$ & \\
\hline
\end{tabular}

Table 2: Olfactory score comparison: preoperatively, 1 month and 3 months postoperatively (Left side) $(\mathrm{N}=45)$.

\begin{tabular}{|c|c|c|c|c|c|c|c|}
\hline \multirow{2}{*}{ Score } & \multicolumn{2}{|c|}{ Preop } & \multicolumn{2}{|c|}{ Postop 1 months } & \multicolumn{2}{|c|}{ Postop 3 months } & \multirow{2}{*}{ P Value } \\
\hline & Mean \pm SD & $95 \% \mathrm{CI}$ & Mean \pm SD & $95 \% \mathrm{CI}$ & Mean \pm SD & $95 \% \mathrm{CI}$ & \\
\hline OIS & $2.56 \pm 1.63$ & $2.07-3.05$ & $3.76 \pm 1.09$ & $3.43-4.08$ & $4.58 \pm 0.62$ & $4.39-4.76$ & \multirow{3}{*}{$<0.001$} \\
\hline OTS & $3.36 \pm 1.71$ & $2.84-3.87$ & $4.6 \pm 1.56$ & $4.13-5.07$ & $5.67 \pm 0.93$ & $5.39-5.95$ & \\
\hline $\cos$ & $5.91 \pm 3.21$ & $4.95-6.88$ & $8.33 \pm 2.41$ & $7.61-9.06$ & $10.24 \pm 1.13$ & $9.9-10.58$ & \\
\hline
\end{tabular}

Table 3: Mean Olfactory score comparison: preoperatively, 1 month and 3 months postoperatively $(\mathrm{N}=45)$.

\begin{tabular}{|c|c|c|c|c|}
\hline \multirow{2}{*}{ Score } & Preop & Postop1 & Postop3 & \multirow{2}{*}{$P$ value } \\
\hline & Mean (SD) & Mean (SD) & Mean (SD) & \\
\hline OIS & $2.64(1.40)$ & 3.71 (1.07) & $4.54(0.51)$ & \multirow{3}{*}{$<0.001$} \\
\hline OTS & 3.19 (1.61) & 4.58 (1.45) & $5.58(0.89)$ & \\
\hline COS & $5.83(2.89)$ & $8.28(2.33)$ & $10.12(1.11)$ & \\
\hline
\end{tabular}

\section{Discussion}

Olfaction impairment is one of the common symptoms of CRS patients. Objective assessment of Olfaction is necessary to know the effect of endoscopic sinus surgery. In present study, the olfaction was assessed in patients undergoing endoscopic sinus surgery in the age group between 18- 50years. In our study about $74 \%$ patients with CRS presented with hyposmia/anosmia preoperatively which significantly affected the quality of life. Similar study conducted by Divyagupta et al. [8] showed that among 40 patients, $70 \%$ had symptoms of hyposmia or of anosmia before surgery. Age group of the patients was between 18 to 50 years, the mean age was 39.82 years and was in the fourth decade. In the 45 patients who participated, $57.78 \%$ were females and $42.22 \%$ were males. Preoperatively $37.78 \%$ were Anosmic, 35.56\% were hyposmic and $24.44 \%$ found to be normsomic. When compared with study by Divyagupta et al. [8] included 40 patients (16 women and 24 men) with a mean age of $32 \pm 9.2$ years. $70 \%$ had olfactory deficit and $30 \%$ were normosmic preoperatively. All patients had undergone preoperative evaluation like nasal endoscopy and CT scan of nose and paranasal sinuses [9-11]. Diagnostic nasal endoscopy with Lund Kennedy grading was done; mean score was found to be $6.73 \pm 2.562$.Similar study conducted by Divyagupta et al[8] the mean nasal endoscopy score was 2.95 preoperatively. CT scans were evaluated using the Lund and McKay scale (maximum score of 24) and the mean score was found to be $13.42 \pm 3$.12.Similar study conducted by Gupta et al. [12] showed preoperative CT score was 11.8. Subjective assessment of olfaction was used earlier, but now the advent of quantitative methods has made olfactory testing accurate. In our study olfaction was assessed objectively using CCCRC Test. Doty et al. $[9,10]$ claimed that the reliability of test was more important factor in the selection of a smell test than the specificity type of olfactory test.

All patients' olfaction were assessed preoperatively, postoperatively at $1^{\text {st }}$ month and $3^{\text {rd }}$ month using olfactory testing tool (CCCRC Test). The mean composite olfactory score was $5.83 \pm 2.89$ preoperatively which increased to $8.28 \pm 2.33$ at 1 
month and $10.12 \pm 1.11$ at 3 months, this difference was found to be statistically significant $(\mathrm{p}<0.001)$. Similar study conducted by Divyagupta et al showed that the mean composite olfactory scores was $2.05 \pm 3.93$ preoperatively, increased to $5.88 \pm 4.56$ at 1 -month and10.43 \pm 4.13 at 3 months. Mean olfactory scores improved postoperatively in all patients. The mean composite olfactory scores improved following endoscopic sinus surgery by 3.5 in Anosmic patients, 2.22 in Hyposmic patients and 1.04 in Normosmic at 1 month. Whereas at 3 month 6.65, 3.22 and 1.95 in Anosmic, Hyposmic and Normosmic patients respectively.

Patients with polyposis had improved, whereas patients without polyposis did not show much change. Similar study conducted by Pade and Hummel [11] did the olfactory testing by using the odor identification test in 206 patients of CRS before and 4 months after FESS surgery. Improvement occurred in $23 \%$ of patients, no change was observed in $68 \%$ and decreased function was seen in $9 \%$. They found greater benefit from surgery in patients with polyposis. Litvack et al. [12] did study on 111 patients with CRS, found significant olfactory improvement in Anosmic patients with nasal polyposis in a 1-year period, but no such change in patients who were without polyposis and Hsu et al. [13] assessed olfactory function in 32 patients, by using the Chinese version of UPSIT, who underwent revision FESS and found improvement in olfactory scores both at 6-12 months and 1-2 years, and also better olfactory improvements in cases with nasal polyposis. Schriever et al. [14] evaluated 113 patients with CRS by using the Sniffin' Sticks test and found no improvement in olfaction in patients without polyposis and significant improvement in patients with polyposis at 3.5 months after endoscopic surgery. And Minovi et al. [15] found patients with nasal polyposis had a higher success rate of olfactory improvement at 6 month follow up period than other patients. In our study no preoperative normsomic patient became an Anosmic following surgery. Study by Hosemann et al. [16] described the preand postoperative results of a qualitative and semi quantitative olfactory function test and demonstrated that no patient whose sense of smell before surgery was normal deteriorated.

\section{Limitations of the study}

In this study, sample size is small. To know accurately the impact of surgery on olfaction, large sample size is required. In our study postoperative follow-up was done till 3 months. It showed Improvement rate in short follow up period. Long term follows up is required as patients assessed within the first couple of weeks after surgery may still be healing from the surgery. Edema and granulation tissue interfere with the results. Conversely patients with initial recovery and significant improvement in early postoperative period later suffer from synechiae formation, recurrence of the disease and new polyp formation in the region of olfactory region which may again have an impact on the olfaction.
In this study olfactory scores in patients with and without polyposis could not be compared statistically because of inadequate size. Postoperative CT scanning is not done in many of patients.

\section{Conclusion}

The evaluation of olfaction in patients undergoing endoscopic sinus surgery shows the improvement in olfactory outcome following the surgery in patients with CRS. 61\%-69\% of CRS patients have a reduced sense of smell [17]. Approximately two thirds of cases are due to the sinonasal disease. Olfactory dysfunction is an increasingly recognized condition with reduced quality of life [18]. Impairment secondary to sinonasal disease is most commonly caused by CRS with or without polyposis. In addition to the mechanical obstruction of odorant to the olfactory cleft caused by polyps and edema, impairment in CRS is also attributed to the inflammatory cytokine mediated olfactory dysfunction. So, the olfactory dysfunction as result of sinonasal disease is unlikely to improve spontaneously without treatment. The olfaction was assessed objectively preoperatively and following surgery at 1 month and 3 months which showed the statistically significant improvement in olfactory scores in all patients. ESS showed significant improvement in olfactory outcome. CCCRCT olfactory test is to perform and helps to assess the outcome of ESS. This test alone does not help to know the postoperative status. In addition to this test anterior rhinoscopy examination and nasal endoscopy assess the surgical outcome more accurately. The study was an attempt to assess the olfaction preoperatively and postoperatively in patients undergoing ESS.

\section{References}

1. Leopold DA, Holbrook EH (2015) Physiology of Olfaction. Flint P, Haughty B, Lund V, Niparko J, Robbins K, et al. (Eds.), Cummings OtolaryngologyHead and Neck Surgery. $6^{\text {th }}$ Ed. Elsevier Saunders, Canada, USA pp. 626627.

2. Doty RL, Bromley SM (2008) Abnormalities of smell. Gleeson M, Browning GG, Burton MJ, Clarke R, Hibbert J, et al. (Eds.), Scott- Brown's Otorhinolaryngology: Head and Neck Surgery. $7^{\text {th }}$ Edn, Hodder Arnold, London, UK pp. 1664-1665.

3. Jund VJ, Jones J (2008) Surgical management of rhinosinusitis. Gleeson M, Browning GG, Burton MJ, Clarke R, Hibbert J, et al. (Eds.), ScottBrown's Otorhinolaryngology: Head and Neck Surgery. $7^{\text {th }}$ Edn, Hodder Arnold, London, UK pp. 1481-1482.

4. Lal D, Stankiewicz JA (2015) Primary sinus surgery. Flint P, Haughty B, Lund V, Niparko J, Robbins K, et al. (Eds.), Cummings Otolaryngology: Head and Neck Surgery. $6^{\text {th }}$ Eds, Elsevier Saunders, Canada, USA pp. 762763.

5. Kimmelman CP (1994) The risk of olfaction from nasal surgery. Laryngoscope. 104(8 Pt 1): 981-988.

6. Veyseller B, Ozucer B (2013) Connecticut (CCCRC) Olfactory Test: Normative Values in 426 Healthy Volunteers: Indian J Otolaryngol Head Neck Surg 66(1): 31-34

7. Gupta D, Gulati A, Singh I, Uma T (2015) Impact of endoscopic sinus surgery on olfaction and use of alternative components in odor threshold measurement. American journal of Rhinology and Allergy 29: 117-120. 
8. Doty RL, McKeown DA, Lee WW, Shaman P (1995) A study of the testretest reliability of ten olfactory tests. Chem Senses 20: 645-656.

9. Deems DA, Doty RL, Settle RG (1991) Smell and taste disorders, a study of 750 patients from the University of Pennsylvania Smell and Taste Center. Arch Otolaryngol Head Neck Surg 117(5): 519-528.

10. Pade J, Hummel T (2008) Olfactory function following nasal surgery. The American laryngological, rhinological and ontological society. The laryngoscope 118: 1260-1264.

11. Litvack JR, Mace J, Smith TL (2009) Does olfactory function improve after endoscopic sinus surgery? Otolaryngology Head Neck Surg 140: 312-319.

12. Hsu CY, Wang YP, Shen PH (2013) Objective olfactory outcomes after revision endoscopic sinus surgery. Am J Rhinol Allergy 27(4): 96-100.
13. Schriever VA, Gupta N, Pade J (2013) Olfactory function following nasal surgery: A 1-year follow-up. Eur Arch Otorhinolaryngol 270: 107-111.

14. Minovi A, Hummel T, Ural A, Draf W, Bockmuhl U (2008) Predictors of the outcome of nasal surgery in terms of olfactory function.Eur Arch Otorhinolaryngol 265(1): 57-61.

15. Hosemann W, Goertzen W, Wohlleben R (1993) Olfaction after endoscopic endonasal Ethmoidectomy. Am J Rhinol 7:11-15.

16. Mohanty S (2016) Effect of Endoscopic Sinus surgery on Olfaction. Clinical Rhinology; An international Journal 9(13): 115-119.

17. Hummel T, Whitcroft KL, Andrews P (2017) Position paper on olfactory dysfunction. Rhinology 56(1): 1-30.

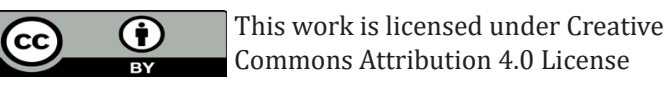

To Submit Your Article Click Here: Submit Article

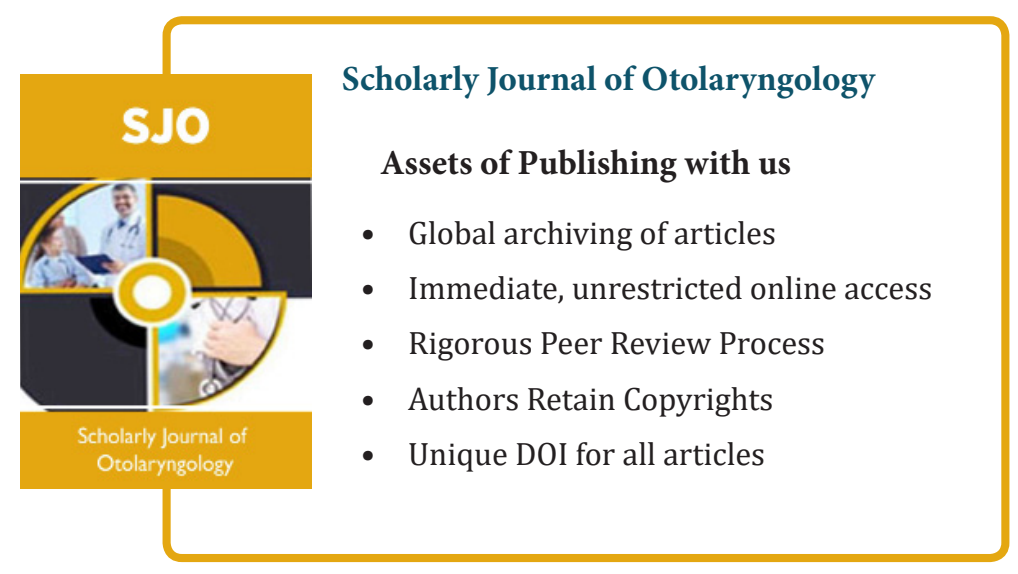

